\title{
Lunar Exploration Architecture Level Key Drivers and Sensitivities
}

\author{
Kandyce Goodliff, William Cirillo, Kevin Earle, J. D. Reeves \\ NASA Langley Research Center \\ Mail Stop 462 \\ Hampton, VA 23681 \\ kandyce.e.goodliff@nasa.gov, william.m.cirillo@nasa.gov, kevin.d.earle@nasa.gov,john.d.reeves@nasa.gov \\ Hilary Shyface, Mark Andraschko, R. Gabe Merrill \\ Analytical Mechanics Associates, Inc. \\ 303 Butler Farm Drive, Suite 104A \\ Hampton, VA 23666 \\ shyface@ama-inc.com, andraschko@ama-inc.com, merrill@ama-inc.com \\ Chel Stromgren \\ Science Applications International Corporation \\ 1710 SAIC Drive \\ McLean, VA 22102 \\ chel.stromgren@saic.com \\ Christopher Cirillo \\ Warwick High School \\ 51 Copeland Lane \\ Newport News, VA 23601 \\ christopher.cirillo@yahoo.com
}

\begin{abstract}
Strategic level analysis of the integrated behavior of lunar transportation and lunar surface systems architecture options is performed to assess the benefit, viability, affordability, and robustness of system design choices. This analysis employs both deterministic and probabilistic modeling techniques so that the extent of potential future uncertainties associated with each option are properly characterized. The results of these analyses are summarized in a predefined set of high-level Figures of Merit (FOMs) so as to provide senior NASA Constellation Program (CxP) and Exploration Systems Mission Directorate (ESMD) management with pertinent information to better inform strategic level decision making. ${ }^{1,2}$
\end{abstract}

The strategic level exploration architecture model is designed to perform analysis at as high a level as possible but still capture those details that have major impacts on system performance. The strategic analysis methodology focuses on integrated performance, affordability, and risk analysis, and captures the linkages and feedbacks between these three areas. Each of these results leads into the determination of the high-level FOMs. This strategic level analysis methodology has been previously applied to Space Shuttle and International Space Station assessments and is now being applied to the development of the Constellation Program point-of-departure lunar architecture. This paper provides an overview of the

\footnotetext{
1 (C)2009 IEEE and United States Government

2 IEEEAC paper \#1310, Version 3, Updated January 7, 2008
}

strategic analysis methodology and the lunar exploration architecture analyses to date. In studying these analysis results, the strategic analysis team has identified and characterized key drivers affecting the integrated architecture behavior. These key drivers include inclusion of a cargo lander, mission rate, mission location, fixedversus-variable costs/return on investment, and the requirement for probabilistic analysis. Results of sensitivity analysis performed on lunar exploration architecture scenarios are also presented.

\section{TABLE OF CONTENTS}

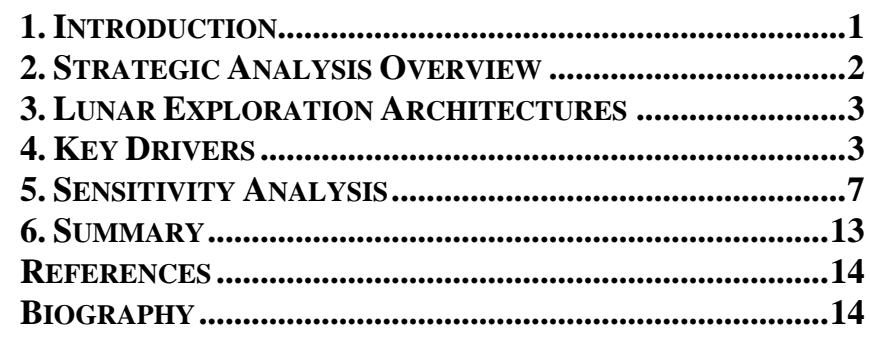

\section{INTRODUCTION}

As NASA and partner agencies, both U.S. and international, move forward with the planning for human lunar return, strategic analysis will play a key role in helping to select architectures and scenarios that will result in productive, reliable, and affordable lunar exploration systems. Strategic analysis serves to inform decision makers of the benefit, viability, affordability, and robustness of system design options by providing 
integrated analysis of system performance over the full system life cycle, from the cradle to beyond the grave. Over the past three years, NASA's Constellation Program (CxP) senior management has applied this strategic analysis methodology to inform the design of lunar transportation and surface architectures, seeking refinement of the NASA Space Exploration Program through ongoing analysis to meet the needs, goals, and objectives defined by NASA's Exploration Systems Mission Directorate in support of the implementation of the Vision for Space Exploration outlined by the President in 2004. Through the application of strategic analysis by the CxP Strategic Analysis Team (SAT) over the last three years, certain key drivers have become apparent. These key drivers contribute directly to the performance, cost, risk, and value of lunar exploration architecture scenarios. In addition, sensitivity analysis is performed on these scenarios to inform the designers of the influence of design choices on the scenarios.

This paper provides an overview of the strategic analysis methodology applied by the CxP SAT. Lunar exploration architectures are briefly described with a focus on overarching assumptions. The key drivers that have been determined to date are discussed, along with how these key drivers affect performance, cost, risk, and value of the architectures. In addition, sensitivity analysis results are discussed.

\section{STRATEgic ANALYSIS OVERVIEW}

In order to achieve NASA's goal of returning to the lunar surface to allow for an extended, permanent human presence, an initial transportation architecture design was developed during the Exploration Systems Analysis Study (ESAS). [1] After the completion of ESAS, a need still existed for high-level study of the integrated system behavior. The CxP Strategic Analysis Team (SAT) was created in response to this need. The SAT supports the development of human lunar architectures through integrated analysis of the performance, cost, risk, and the value of the lunar transportation and surface systems. The strategic analysis methodology employed by the SAT allows NASA senior-level decision makers to understand the benefit, viability, affordability, and robustness impacts of various system design options. This strategic level analysis methodology has been previously applied to Space Shuttle and International Space Station assessments.

Strategic analysis focuses on integrating performance, affordability, and risk analysis, and capturing the linkages and feedbacks between these three areas. This strategic level analysis capability does not include element design and sizing capability but rather performs resource utilization analysis with the use of predefined lunar transportation and surface system element options.
Specific performance data for lunar exploration architecture element options are imported into a library from expert-driven design/sizing tools or expert analysis. The SAT performs a high-level analysis that evaluates lunar exploration architecture scenarios through deterministic and probabilistic manifesting, risk analysis, affordability analysis, and value evaluation. [2, 3]

Scenario analysis is completed for a planned set of missions in which cargo and crewed landers deliver elements, logistics, and humans to the lunar surface. Each type of lander has a capacity that is available to deliver elements and/or logistics. The scenario analysis methodology begins with the development of a deterministic manifest that is "closed." Scenario closure is achieved when the elements, logistics, and carriers can be manifested within the available capacity of the transportation system to support the crew surface stay duration for each mission. [4] Deterministic analysis focuses on when and where the logistics and elements are delivered according to two initial optimization variables. The primary analysis metric captures each scenario's planned cumulative crew surface duration. The secondary variable, unallocated delivery capacity, serves to differentiate between scenarios that share similar crew surface durations. Unallocated delivery capacity is lander capacity that is not required to support mission requirements and can be used during scenario implementation to increase productivity, reduce costs, or mitigate risks.

Risk analysis evaluates the safety and uncertainty of a scenario. This method uses an event tree to simulate all possible failures. The event tree supplies information to determine the probability of a failure for each mission. Failure event consequences can range from the loss of crew to the loss of an element or multiple elements. The current risk model focuses on the vehicle reliability of the transportation system. A lunar surface system model will be included in the near future.

After the deterministic scenario has been created, the scenario is analyzed probabilistically. Probabilistic analysis involves the use of the deterministic scenario and the risk analysis as a starting point for a Monte Carlo analysis. The probabilistic tool analyzes the scenario on a mission-by-mission basis and stochastically predicts whether that particular mission will experience a failure. If the mission fails, then the tool analyzes how that failed mission will affect the rest of the scenario and remanifests the remaining missions appropriately. If the mission succeeds, then the tool continues on to the next mission and continues the analysis process until all of the missions in the scenario have been analyzed. The probabilistic analysis allows a determination of the robustness of the scenario by accounting for the uncertainty and the inherent risks of human space flight. 
Affordability analysis is also completed by starting from the deterministic scenario. Affordability analysis incorporates the life cycle cost (LCC) of various lunar exploration elements, integrates these element costs, and compares the integrated costs to projected budgets. The main driving parameters that affect the affordability analysis are scenario definition and element manipulation, budget scenarios, and the cost savings from participation of international and commercial partners.

The data and conclusions from these various analyses are consolidated into high-level figures of merits (FOMs). These FOMs are intended to help inform the decision makers of the merits of various scenario options in a comparative form. FOMs measure the affordability, the extensibility and experience, the science and lunar survey, the sustainability, and the risk for a scenario.

\section{LUNAR EXPLORATION ARCHITECTURES}

Following the completion of ESAS, NASA has continued to explore the trade space for lunar exploration architectures, including both transportation systems and surface systems. Efforts have been conducted through the Lunar Architecture Team (LAT) and Constellation Architecture Team (CxAT) studies and efforts from various Constellation Program offices. The LAT Phase 1 study [5] (2006) initiated a broad assessment of lunar architecture options that led to the selection of a South Pole Lunar location to support the development of a permanent outpost. The LAT Phase 2 study [6] (2007) determined the need for a diversified lunar lander system that provides both a dedicated crewed lander and cargo lander. The CxAT Lunar study identified the need for an enhanced Ares V/Earth Departure Stage system to support global access with an anytime return (2008).

Throughout these studies, the focus of the lunar exploration architecture options has been on the buildup of an outpost at the lunar South Pole with Human Lunar Return (HLR) in 2019. The flight rate has been varied from two to four missions per year. The crew size of four to the lunar surface has remained constant throughout the studies. Primary objectives have included the achievement of continuous human presence on the lunar surface, as well as delivering science, internationalpartner, commercial, and public engagement payloads to the lunar surface. As these studies have progressed, assumptions have changed which have led to the discovery of certain key architecture drivers. These key drivers, which will be discussed in Section 4, have a significant impact on performance, cost, risk, and value of the lunar exploration architecture options.

The decision to establish an outpost with continuous human presence has resulted in the development of a lunar surface infrastructure design and logistics resupply methodology that meets system requirements given the current lunar transportation system design. The lunar surface infrastructure typically accounts for 30 to 40 percent of the total delivered cargo. The remaining 60 to 70 percent of the cargo delivered is the logistics and associated carriers to enable the sustained human presence. Logistics consists of pressurized goods (i.e., crew consumables and spares and maintenance), unpressurized spares \& maintenance, gases, and liquids that are required to support crewed operations and maintain surface asset functionality. For these types of exploration architectures, logistics account for approximately 25 to 33 percent of the total mass delivered to the lunar surface. In addition to the logistics, cargo mass of the logistics carriers accounts for approximately 25-33percent of the total delivered mass. Figure 1 shows a typical breakdown of the primary categories of goods that are required to support a human lunar outpost in these exploration architectures.

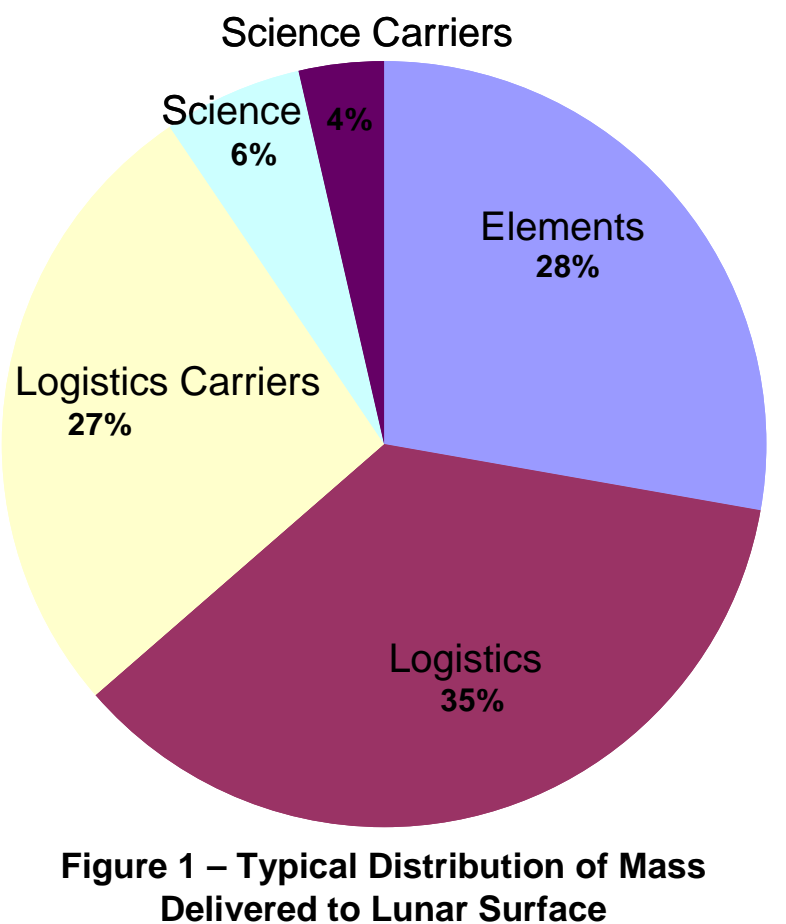

\section{KEY DRIVERS}

The analysis that has been performed over the past several years has demonstrated certain patterns relative to key factors that play a recurring significant role in affecting overall architecture performance, cost, and risk when compared with an associated return on investment. To date, observed architecture level key drivers include (1) augmentation of a crew delivery capability with a dedicated cargo lander system, (2) sustaining a proper annual mission rate, and (3) supporting the capability to access a wide range of lunar surface locations. 
An analysis of the projected behavior of the complex interactions between the lunar transportation systems and the lunar surface systems has demonstrated that evaluation of the planned performance of the integrated system alone is not sufficient to adequately inform senior NASA management of the relative merit of different options. The analyst must assess the impact that the reliability and the uncertainty of these systems have on performance. Therefore, both the deterministic and the stochastic behavior of the architecture are represented in the resulting data sets.

Finally, current study results indicate that one must perform sufficient sensitivity analyses for each architecture option to ascertain the additional return on investment that may be realized with a minimal additional amount of budget resources. This type of analysis approach defines the relatively large return on investment that can be obtained with a small number of additional "variable" dollars, when compared with the large "fixed" amount of funding that is required to achieve even a minimal return on investment.

Thus, both the independent variables explicitly used in the analysis, as well as the interpretation of the data results themselves, become key drivers in terms of making properly informed architecture level strategic decisions.

The results that are included in the following subsections are examples of measures of performance, cost, risk, and value and are not the complete set of FOMs (as documented in references 2 and 3). Each of these metrics should be considered when determining how lunar exploration architecture scenarios compare with one another. For the purpose of this paper, a subset of these measures has been chosen to demonstrate how scenarios are compared and why the following subsection topics are key drivers.

The cases evaluated are all assumed to have a fixed end date of FY30 to allow for a quantitative comparison of scenarios. The performance is measured as the cumulative number of surface stay days that the crew can live on the lunar surface. The cost is measured as a normalized cost through FY20 and FY30. The risk is measured as a normalized probability of loss of crew (PLOC) over the total number of missions within a scenario. The cost and risk data are normalized as a result of the sensitive nature of the data. The value is measured as the percentage of extensibility objectives that are met and the amount of science mass that is delivered to the lunar surface over the campaign [2]. The extensibility objectives are a set of technology demonstration objectives that can be demonstrated on the Moon to support future Mars exploration.

\section{Inclusion of Cargo Lander}

LAT Phase 1 focused on the delivery of goods to the lunar surface with crewed lunar landers. However, as past programs such as Apollo have shown, human-rated spacecraft tend to increase in mass between conceptual design and the manufacturing of the vehicle. This behavior is compounded by the multiple components of an integrated transportation system. Growth in one of these components will affect the performance and growth of the other components. In addition, architecture scenarios where the goal is to achieve continuous human presence require large quantities of logistics and generally large elements to be delivered to the lunar surface to support a sustained presence. It is challenging to design a crewed lunar lander with the capability to deliver a large enough payload to support continuous human presence along with the capability to deliver the crew. Therefore, cargo landers should be considered to augment the crewed lunar lander. Cargo landers allow for robustness against crewed lander performance and element mass growth.

Figure 2 presents a comparison between two sample scenarios, one with a cargo lander and one without a cargo lander. The total number of flights and the elements delivered to the lunar surface for each of the scenarios are the same; hence, the total cost through FY30 is essentially the same, with some slight variability due to a change in the number of cargo flights, which incur slightly lower costs than the crewed flights.

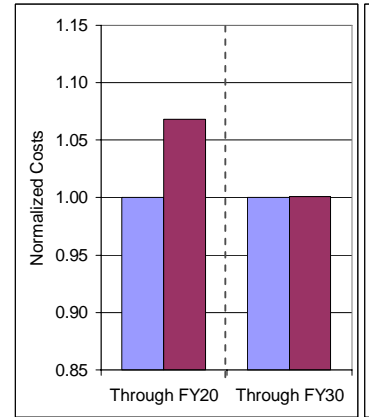

No Cargo Lander
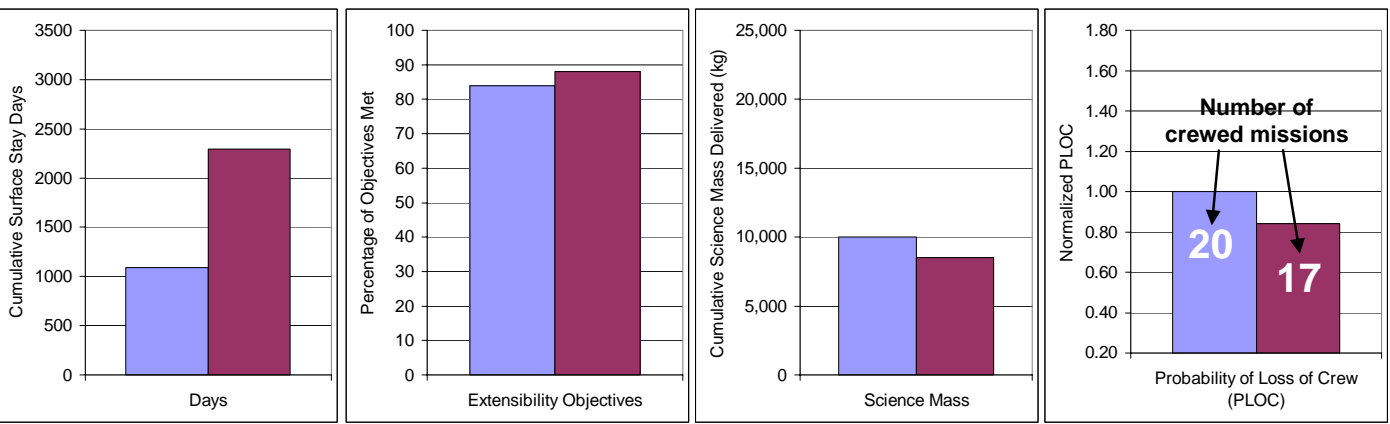

$\square$ Cargo Lander

\section{Figure 2 - Cargo Lander Comparison}


The cost for the cargo lander scenario is slightly higher through FY20 because key elements are delivered significantly earlier, moving the developmental costs of these elements earlier in the program. The cumulative number of surface stay days is increased by 120 percent because the elements and logistics to support the crew can be delivered earlier and in larger quantities, thus allowing for longer duration stays on the lunar surface through FY30. The percentage of extensibility objectives that are met is only slightly increased because of the increased surface stay days but remains similar because the same elements are delivered and the identical objectives are completed for each scenario; however, the objectives are satisfied earlier in the cargo lander scenario. The science mass allocation is slightly higher for the no cargo lander scenario, driven by the assumption for these scenarios that the science mass delivered is a function of the number of crewed missions to the lunar surface. The probability of loss of crew (PLOC) is slightly lower for the cargo lander scenario because fewer crewed missions exist in the scenario and additional uncrewed flights earlier in the scenario increases vehicle maturity.

The inclusion of cargo landers into lunar exploration architecture scenarios mitigates potential reduction in the transportation system delivery capacity. Cargo landers benefit architecture scenarios regardless of crewed lander capacity, but the inclusion of cargo landers has a more pronounced effect as crewed lander capacity decreases. The inclusion of cargo flights within a scenario can provide robustness against transportation system uncertainty because of the extra performance capability of a cargo lander. Any scenario that requires the maximum payload capability will always lack robustness with respect to uncertainty in performance capability.

\section{Mission Rate}

The impact of varied mission rates from one to four missions per year, with a varied crewed and cargo lander ratio, has been studied and is a key driver of system performance. A mission consists of either a crewed launch (Ares I launch + Ares V launch) or a cargo launch (Ares V launch). Assumed mission rates drive all phases of a lunar exploration architecture scenario, including the outpost buildup phase, the outpost operational phase, and any planned sortie phase. Two variations of scenarios have been explored: those that have a constant number of missions per year and those with a variable number in the early years and a steady-state rate later in the scenario. For the example shown in Figure 3, a constant number of yearly crewed and cargo missions were explored (see Table 1). These cases have identical elements delivered to the lunar surface; no sortie missions were included for the purposes of this comparison.

Table 1. Mission Rate Comparison

\begin{tabular}{|c|c|c|c|}
\hline $\begin{array}{c}\text { Number of } \\
\text { Crewed } \\
\text { Missions }\end{array}$ & $\begin{array}{c}\text { Number of } \\
\text { Cargo Missions }\end{array}$ & $\begin{array}{c}\text { Total Number } \\
\text { of Missions }\end{array}$ & $\begin{array}{c}\text { Total Number } \\
\text { of } \\
\text { Launches }\end{array}$ \\
\hline 1 & 1 & 2 & 3 \\
\hline 1 & 2 & 3 & 4 \\
\hline 2 & 1 & 3 & 5 \\
\hline 2 & 2 & 4 & 6 \\
\hline 3 & 1 & 4 & 7 \\
\hline
\end{tabular}

As seen in Figure 3, the cost through FY20 for the mission rate cases generally increases as the cargo lander flight rate increases because the surface elements can be delivered earlier within the scenarios, thereby placing the design, development, test and evaluation (DDT\&E) costs earlier. Through FY20, cost increases when the number of crewed lander missions increases because of the cost of the additional missions. This cost increase also is noticeable when costs are compared through FY30, because the bulk of the additional cost is directly attributed to the increased launches per year. The cumulative number of surface stay days increases as missions are added; the largest number of surface stay days is achieved when two crewed and two cargo missions are flown per year. This big increase results from the balance between the logistics that are required to support the crew for extended durations and the delivery capacity of the two cargo landers. However, the decrease in cumulative surface stay days results for the ' $3 \mathrm{crew}$ 1cargo' case because launching one cargo mission per year does not provide a balance between cargo lander

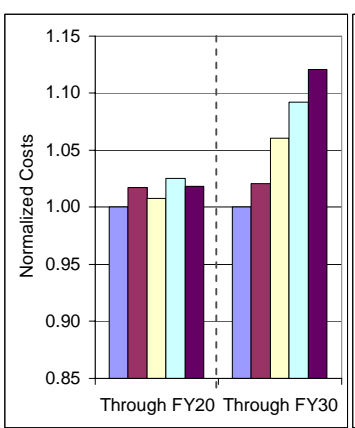

1crew 1cargo

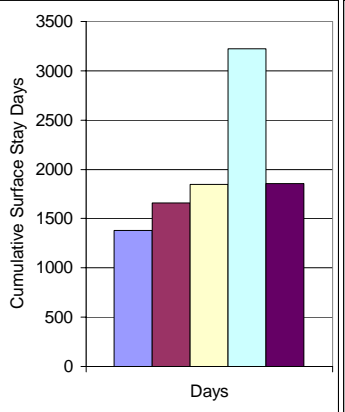

1crew 2cargo

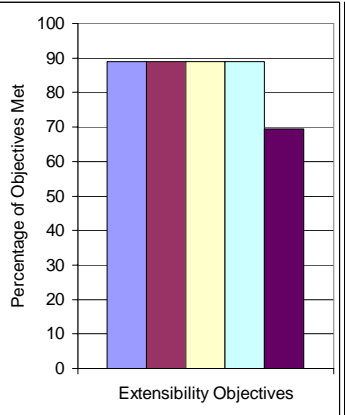

2crew 1cargo

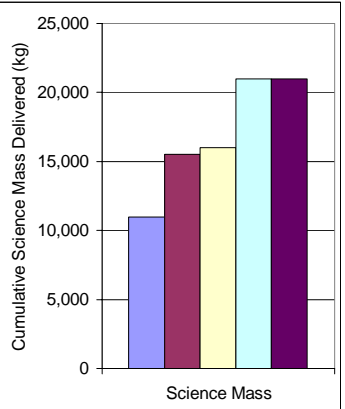

2crew 2cargo

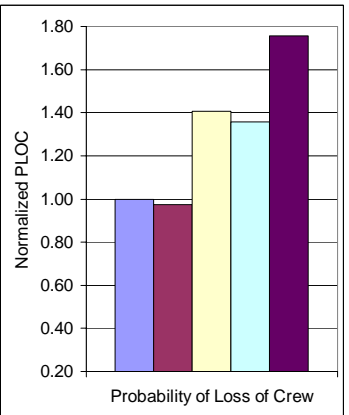

3crew 1cargo

Figure 3 - Mission Rate Comparison 
capacity and crew logistics requirements for extended durations.

The percentage of extensibility objectives that are met is similar among all cases, except for the '3crew 1cargo' case. This exception is mainly driven by the inability to achieve continued human presence as a result of the lack of available cargo space to send logistics to support crews for long-duration missions. The cumulative science mass increases as the number of flights increases because the assumption is made that each lander delivers a fixed amount of science. The PLOC increases as the number of crewed missions increases and decreases as the number of cargo missions increases. Again, this effect results from the fact that cargo missions do not affect the PLOC calculation but contribute to the maturity growth of the launch vehicle.

Although the lower flight rate cases cost less and are less risky, they have less performance when compared with cases with a higher flight rate. As mission rates are varied, a balance between crewed and cargo flights must be maintained to achieve a high return on investment.

\section{Mission Location}

As stated in the publication by the National Research Council (NRC), “The Scientific Context for Exploration of the Moon" [7], maximizing the number of locations visited on the lunar surface would be the primary way to maximize scientific return. Based on this recommendation by the NRC, sortie missions to four alternate lunar locations were explored; these sortie missions replaced planned crewed missions to the Outpost.

For the mission location comparison, the '2crew 1cargo' and '2crew 2cargo' cases from the mission rate section are used. Four sortie missions were added to each of these scenarios. A comparison of these scenarios is shown in Figure 4 . The cost of cases with sortie missions is slightly less than the cost of scenarios without sortie missions. This cost reduction is mainly attributable to the reduction of logistics required to support the crew while at the Outpost. The number of cumulative surface stay days decreases for scenarios with sortie missions because the number of missions through FY30 remains the same, but the Outpost missions that have been eliminated have longer durations than the assumed seven-day sortie missions. The percentage of extensibility objectives met is also reduced for scenarios with sortie missions because Outpost missions are replaced with sortie missions and the extensibility objectives are Outpost focused. The science mass that is delivered is identical given that the science mass is a function of mission rate, not location. If science mass to the Outpost was a metric, then cases with sortie missions would obviously result in a lower cumulative mass; however, given the NRC recommendation, scientific return is maximized by visiting multiple locations. The PLOC is also identical because this value is currently based on the mission rate and the number of crewed missions.

\section{Planned Versus Expected}

Strategic analysis is typically done in a deterministic manner in which scenario performance is optimized over a set of missions that proceed exactly as planned. However, given the large risks that are involved with human space flight and the high level of uncertainty in technology, budget, vehicle reliability, and schedules, it is important to characterize the possible range of behavior of the system by performing probabilistic analysis. [8] For this analysis, probabilistic results were produced based solely on the reliability of the transportation system. These transportation system reliabilities are derived from preliminary risk analysis study results provided by the individual Constellation transportation projects (i.e., Orion, Ares I, Ares V, and Altair) for incorporation into an integrated risk model developed by the SAT. These individual transportation project reliabilities are then further parsed by mission phase to allow for proper accounting of mission specific failure consequences. These differences between deterministic and probabilistic results would likely become greater if lunar surface system reliabilities and/or uncertainty in technology development schedule are also taken into account.
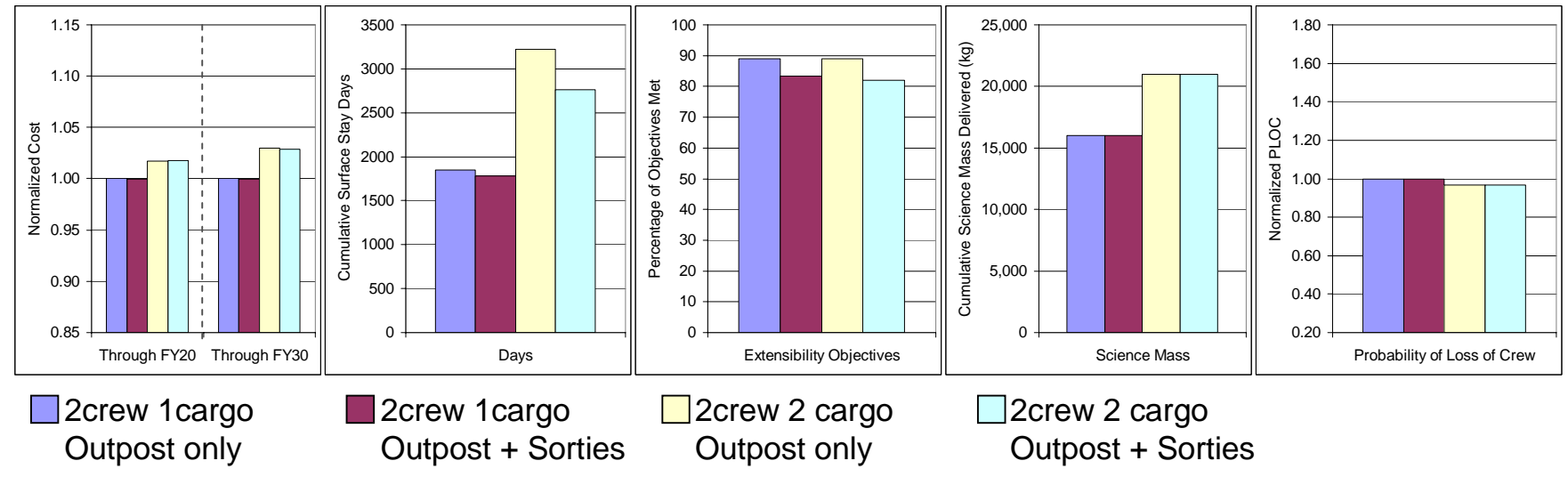

2crew 1cargo

$\square$ 2crew 2 cargo

2crew 2 cargo

Outpost + Sorties

Outpost only

Outpost + Sorties

Figure 4 - Mission Location Comparison 


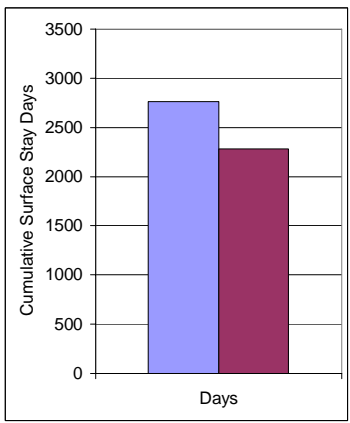

Deterministic

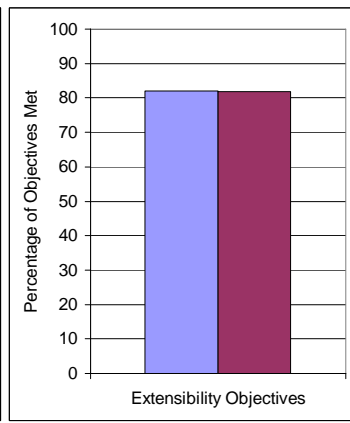

Probabilistic
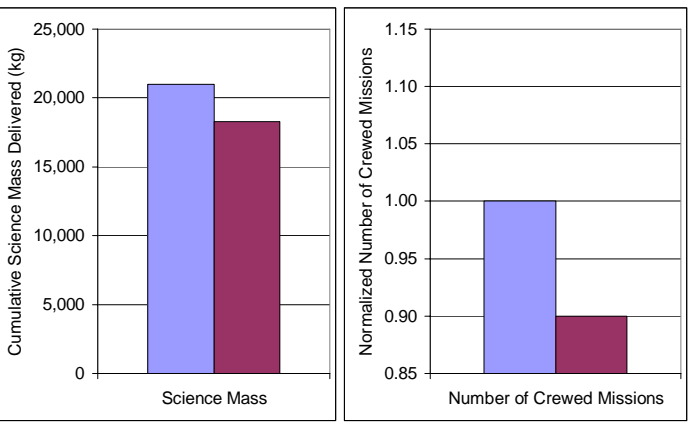

Figure 5 - Comparison of Deterministic and Probabilistic Scenario Analysis

Within this analysis, probabilistic results are expressed as the mean value produced though Monte Carlo analysis. These values represent an average of expected outcomes that account for real-world uncertainties driven by transportation system failure rates for lunar missions. This characterization of the difference between the planned and expected outcomes is a key driver as one must overplan from the beginning in order to achieve the planned end state. Typically, the expected end state will yield a lower return on investment than the initially planned value. If the planned return on investment is required, then analysis indicates that either additional resources in the form of increased flight rate and/or schedule will be required to meet original objectives. For the '2crew 2cargo' case presented above, the deterministic and probabilistic results are presented in Figure 5.

As quantified in this figure, the cumulative surface stay days, science mass, and number of crewed missions are all decreased for the probabilistic results, as compared with the planned values. The probabilistic values are all limited because the scenarios end in FY30. If the probabilistic scenario was allowed to progress past FY30, continuing until the original planned missions were completed, the metrics would be the same as the deterministic results. The percentage of extensibility objectives met is approximately the same because most of the objectives are met within a given number of flights, which are completed in both scenarios. This type of uncertainty information is extremely important to understand early in the design process so that limited program resources can be spent to tackle the more important problems.

\section{Return on Investment - Fixed Versus Variable Cost}

For assessment of return on investment, the mission rate cases (see Table 1) were used. Independent of which scenario is chosen, a certain "fixed" amount of investment in lunar transportation systems and lunar surface systems is required to enable even the simplest option (i.e., '1crew 1cargo'). Additionally, the analysis results indicate that a much larger return on investment can be achieved when a relatively small amount of additional "variable" funds are used to increase the annual mission rate. This return on investment is measured in cumulative surface stay days and is illustrated in Figure 6(a).

However, one must also take into account other measures to determine the best overall return on investment. For example, Figure 6(b) shows the cumulative surface stay days versus the normalized PLOC for the cases. The '2crew 2cargo' case, as shown in Figure 6(b), does have an increase in PLOC over the '1crew 1cargo' case but allows for a significant number of surface days compared with the other cases. The two graphs in Figure 6 illustrate how combining multiple metrics can aid decision makers in making more informed decisions.

\section{SENSITIVITy ANALYSis}

Sensitivity analysis facilitates the understanding of parameters and their impact on performance, cost, risk, and value. As the goals and assumptions of the lunar exploration architectures are modified, sensitivity analysis is performed to determine the robustness of these architectures. The results presented in this section may or may not translate to future architectures as the ground rules and assumptions are updated. Sensitivities that result in the same trends regardless of assumptions and ground rules typically lead to the discovery of key drivers.

Sensitivity analysis is typically applied to deterministic scenarios to understand the implications of given assumptions at a higher level of analysis. The primary method to achieve closure in the sensitivity analyses is to increase or decrease crew surface days. The specific elements for all of the sensitivity analyses were not resized to optimize the scenario. In general, the delivery order of the elements was also maintained. However, in some cases elements had to be reordered to allow sufficient logistics to be delivered to support the crew. To fully take advantage of (or mitigate) a change in 


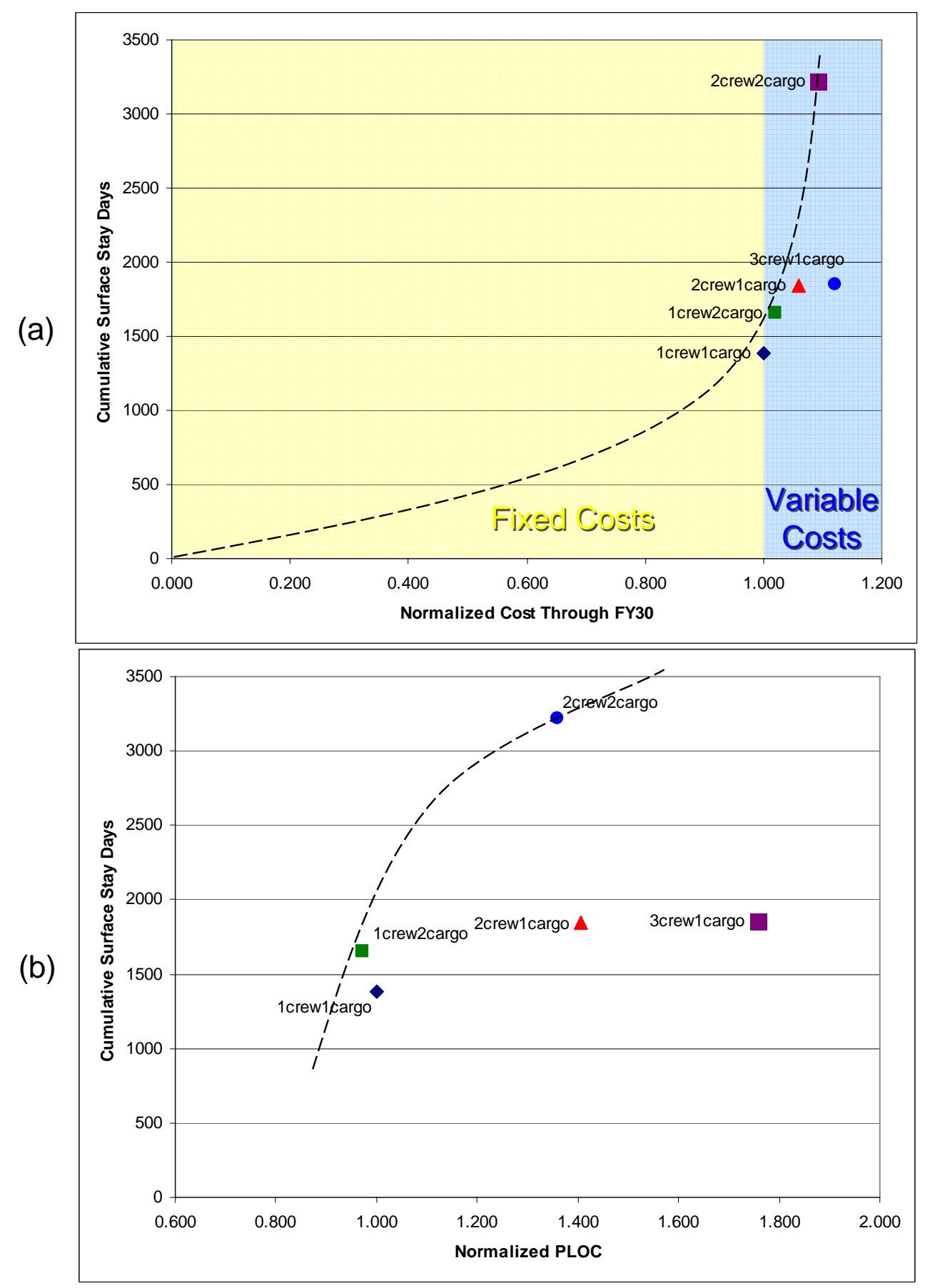

\section{Figure 6 - (a) Fixed Versus Variable Costs \\ (b) PLOC Versus Cumulative Surface Stay Days}

performance, the scenario should be fully restructured, with the elements being resized to optimize the overall performance of the scenario.

The primary initial optimization variable that was used to compare sensitivity cases was planned cumulative surface stay days. The secondary variable was unallocated lunar lander capacity over the entire scenario. Often, scenarios are distinctly different but have similar planned cumulative surface stay days. This secondary variable was used to differentiate between those scenarios. The unallocated lander mass cannot be used to increase cumulative surface stay days because of the inherent constraints on the scenario. The sensitivities discussed below include lander cargo capacity, pressurized volume, technology options, spares and maintenance, element mass, and logistics carrier capacities. 


\section{Cargo Capacity}

The lander capacity sensitivity explores how various lander capacities affect the ability to deliver crew and cargo to the lunar surface. For both the crewed and cargo landers, an ideal available mass exists that will efficiently carry all elements and required logistics and spares for the missions. The cargo lander capacity was incrementally changed by 0.5 metric tons (t), positively and negatively. The crewed lander capacity remained at $1 \mathrm{t}$ for outpost missions and $0.55 \mathrm{t}$ for sortie missions. The crewed lander capacities were not varied because the sensitivity analysis is exploring the performance of the lander, not the design of the lander. It is assumed that the design of the lander would change to retain the crewed lander capability at the expense of the cargo lander performance.

Figure 7 quantifies the results of the sensitivity analysis. From the baseline case, decreasing the lander capacity dramatically reduces crew surface duration and available unallocated lander capacity. Increasing the lander capacity minimally beneficially affects crew surface duration, yet the available unallocated lander capacity increases significantly.

The cargo lander capacity drives early missions (i.e., missions thru 2024). As denoted in the white boxes in Figure 7, the lower mass that is available to the missions early on results in Outpost elements being pushed back to later missions and the period of Outpost buildup being extended. The higher lander capacity, as previously stated, carries sufficient unallocated mass capacity to afford a mission of opportunity. A mission of opportunity results from sufficient unallocated lander capacity existing such that a cargo mission could be replaced by a crewed mission without impacting the scenario's cumulative crewed surface duration. Missions of

opportunity can be used to deliver additional infrastructure, add a sortie mission to an alternate lunar location, and reduce the flight rate to the Moon in preparation to transition to Mars.

\section{Pressurized Delivery Volume}

Pressurized carriers, with volume and mass capability constraints, are required to carry the pressurized logistics that are necessary for surface operations. Because the available pressurized volume and mass can vary with assumed sizing of these pressurized carriers, the sizing affects the amount of pressurized crew consumables that can be carried. Restricting the crew logistics delivery capability constrains the crew surface duration.

The volume and mass of the pressurized carriers are dependent on lander capacity. Often when insufficient mass capacity is available on the lander, plenty of pressurized volume is available, or vice versa. Because both quantities are constraints to having a scenario "close", balancing the two quantities is a necessity. The trade-off between lander capacity and pressurized mass stems from elements able to carry pressurized logistics within it. Elements with pressurized capability, such as a habitat or a dedicated pressurized logistics carrier, require other elements to function-power, communications, mobility, and so on. These additional elements use the lander mass capacity, which leaves little room for any additional mass. These are typically early missions in the scenario when the focus of the scenario is outpost buildup; thus, the early cumulative mission days are kept short. The other aspect is generally represented in missions later in the scenario. Here, crew surface time is limited by the pressurized consumables that are available. After the Outpost elements are delivered, cargo missions become resupply missions for restocking the spares and logistics. Without the large

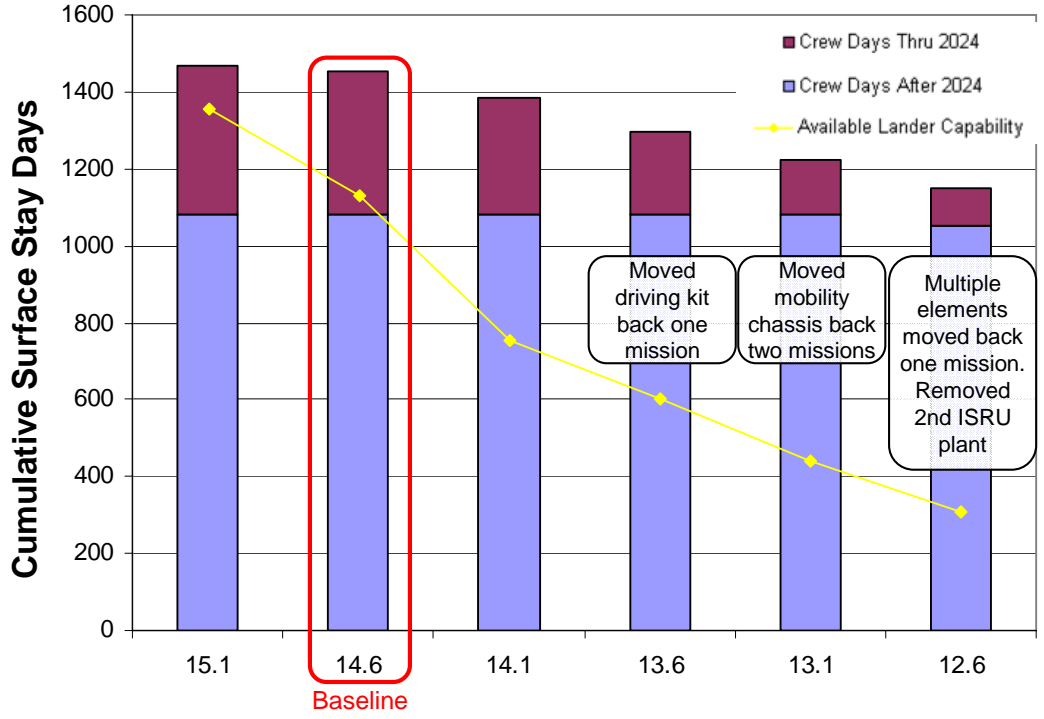

Cargo Lander Capacity ( $\mathrm{t}$ )

Figure 7 - Lander Capacity Sensitivity

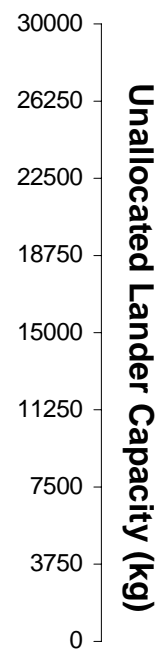
mass elements, excess lander capacity is available. The pressurized volume and mass is restricted to the size of a pressurized logistics module (PLM); because of lander volume constraints, typically only one PLM is delivered per cargo mission.

Figure 8 shows examples of scenarios that are limited in lander capacity and pressurized mass/volume. The graphs in Figure 8 (a) and (c) show the pressurized volume utilization. The green bars indicate how much of the available volume is used, and the yellow bars indicate how much is not used. 
(a)
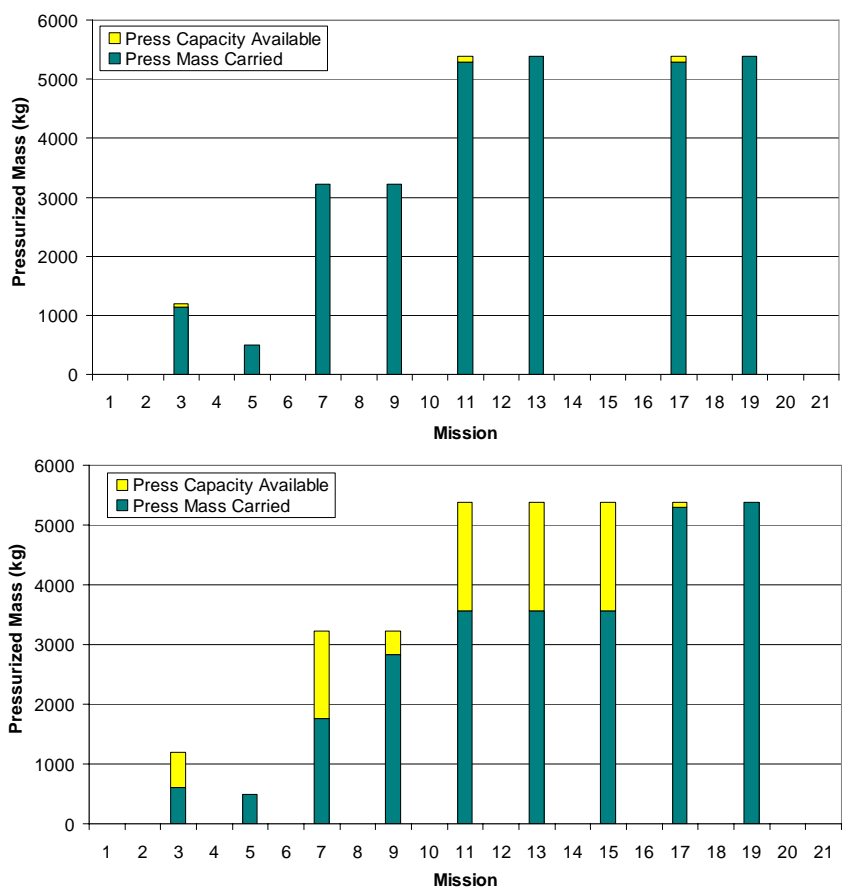

(c) (b)
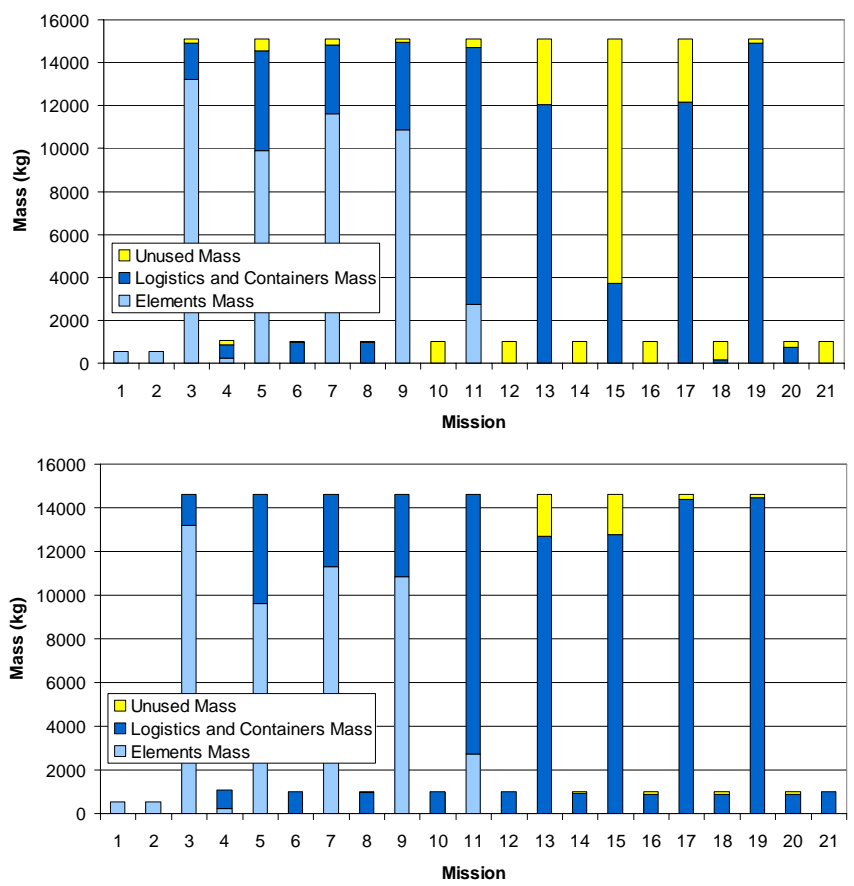

(d)

Figure 8 - Example Cases of: (a) \& (b) Total Capacity Limited Scenario (c) \& (d) Pressurized Volume Limited Scenario

The graphs in Figure 8 (b) and (d) show the capacity that is used for elements (light blue), for logistics and carriers (dark blue), and the unused mass (yellow). The top two graphs show a scenario that is limited in lander capacity. The bottom two graphs show a scenario that is pressurized volume limited.
The sensitivity analysis completed for pressurized carriers varied the volumetric size, affecting the mass-carrying capacity of a PLM. The sensitivity analysis was executed by varying the diameter and the length of a PLM. These dimensional changes increase or decrease the pressurized capacity and allow for more or fewer pressurized logistics delivered to the lunar surface. Larger useable pressurized

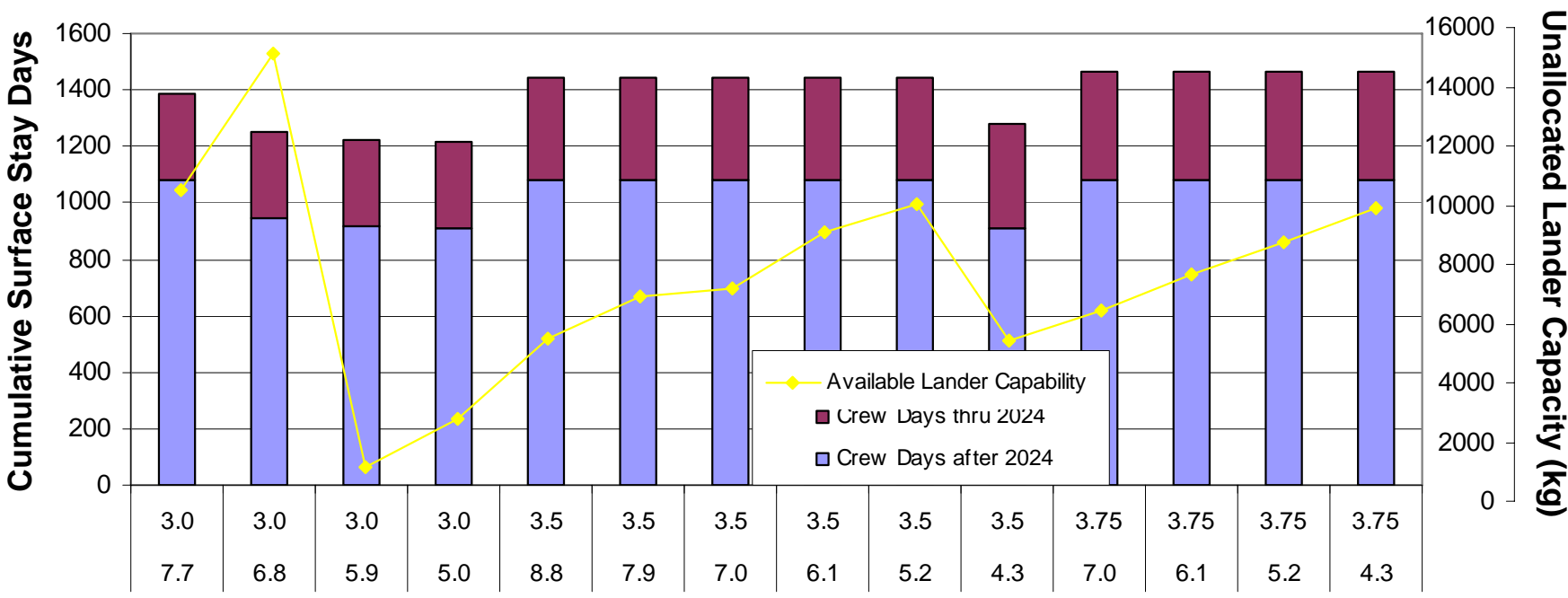

PLM Size - Diameter $x$ Length $(\mathrm{m})$

Figure 9 - Pressurized Logistics Module Sensitivity 
capacities allow for extending crew surface stay duration, whereas smaller useable pressurized capacities will reduce surface stay duration unless multiple pressurized carriers can be delivered on a cargo lander.

Figure 9 shows the results of varying the dimensions of a PLM. The graph illustrates that an increase in diameter results in an increase in crew surface stay duration; however, the increase in diameter from $3.5 \mathrm{~m}$ to $3.75 \mathrm{~m}$ does not significantly increase the surface duration. In addition, an increase in length typically decreases the available lander capacity.

\section{Technology Options}

Application of advanced technologies can allow for mass savings over the duration of a scenario. However, technology does come at a cost, usually in dollars and schedule. The technology options that were considered in this sensitivity include Environmental Control and Life Support System (ECLSS), In-Situ Resource Utilization (ISRU), lander water scavenging, and Extra Vehicular Activity (EVA) water recovery. Each phase of the scenario has a different set of requirements on how each of these technology options is used.

ECLSS involves water recovery, carbon dioxide $\left(\mathrm{CO}_{2}\right)$ reduction, brine recovery, drying of solids, and laundry usage. For water recovery, all cases assume that 93.5 percent of the water is recovered. For $\mathrm{CO}_{2}$ reduction, a Sabatier reactor is used, which is a methane reduction method. For brine recovery, the percentages are varied at 99 percent, 50 percent, and 0 percent. With solids drying, recovery options are 0 percent and 50 percent. Laundry usage is either on or off, to differentiate between whether the crew washes their clothes or brings all of the clothes that are needed for the duration of the mission. The ECLSS is delivered as part of the main habitat element and does not start functioning until the habitat element is delivered on the lunar surface. The laundry machines are

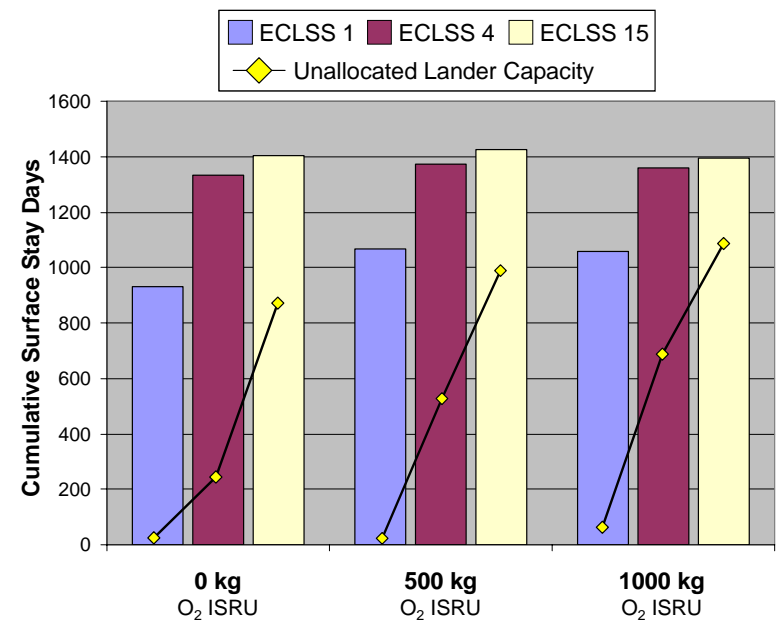

No Water Scavenging also delivered in the main habitat element, but laundry usage does not begin until a crewed mission surface stay duration exceeds 21 days, a breakpoint at which the water required for laundry is less than the clothes mass with no laundry usage. The oxygen production ISRU plant capability was varied between $0 \mathrm{~kg}, 500 \mathrm{~kg}$, and $1000 \mathrm{~kg}$ per year. Lander water scavenging assumes usage of the leftover fuel from the lander, and water is generated from it to produce either $200 \mathrm{~kg}$ or $400 \mathrm{~kg}$ of water per lander delivered to the lunar surface. EVA water recovery involves recovering water back from the EVA suit. These case combinations are shown in Figure 10 and are defined as:

- ECLSS 1: 0 percent water recovery, 0 percent brine, 0 percent solids, no $\mathrm{O}_{2}$ generation, no $\mathrm{CO}_{2}$ recovery, no $\mathrm{EVA} \mathrm{CO}_{2}$ recovery

- ECLSS 4: 93.5 percent water recovery, 0 percent brine, 0 percent solids, $\mathrm{O}_{2}$ generation, no $\mathrm{CO}_{2}$ recovery, no EVA $\mathrm{CO}_{2}$ recovery

- ECLSS 15: 93.5 percent water recovery, 99 percent brine, 0 percent solids, $\mathrm{O}_{2}$ generation, $\mathrm{CO}_{2}$ recovery $\left(\mathrm{CH}_{4}\right)$, no EVA $\mathrm{CO}_{2}$ recovery.

Trends show that water scavenging can boost the length of crew surface stay duration by a considerable number of days and also can free up lander capacity. The analysis also shows that water scavenging limits the impact of an ISRU plant (500 kg per year) to a slightly increased crew surface stay duration and that a second ISRU plant (1000 $\mathrm{kg}$ total per year) has no effect on the crew surface stay duration. As the ECLSS becomes more "closed" by the assumed technologies and with water scavenging available, ISRU has a minimal impact on the unallocated lander capacity as well. An ECLSS technology investment greatly increases the crew surface stay duration compared with a completely open ECLSS.

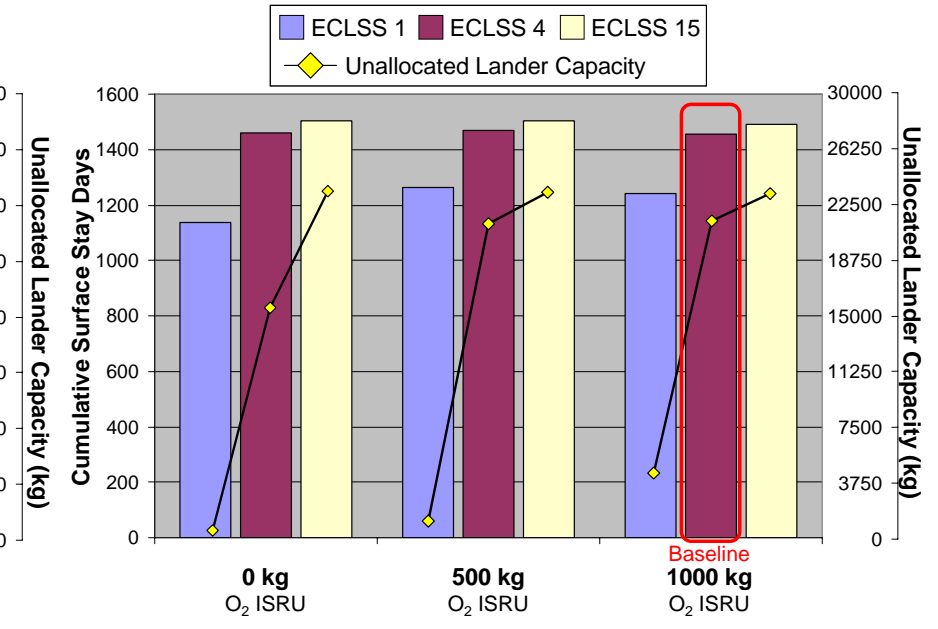

Water Scavenging

Figure 10 - Comparison of Technology Options 


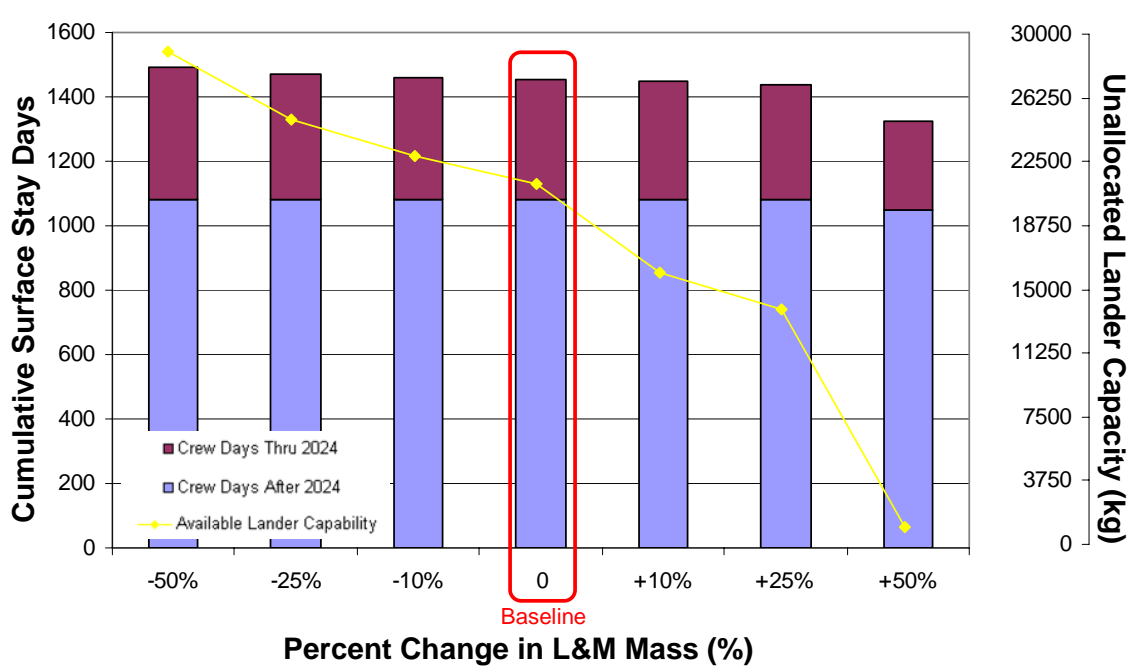

Figure 11 - Spares and Maintenance Mass Sensitivity

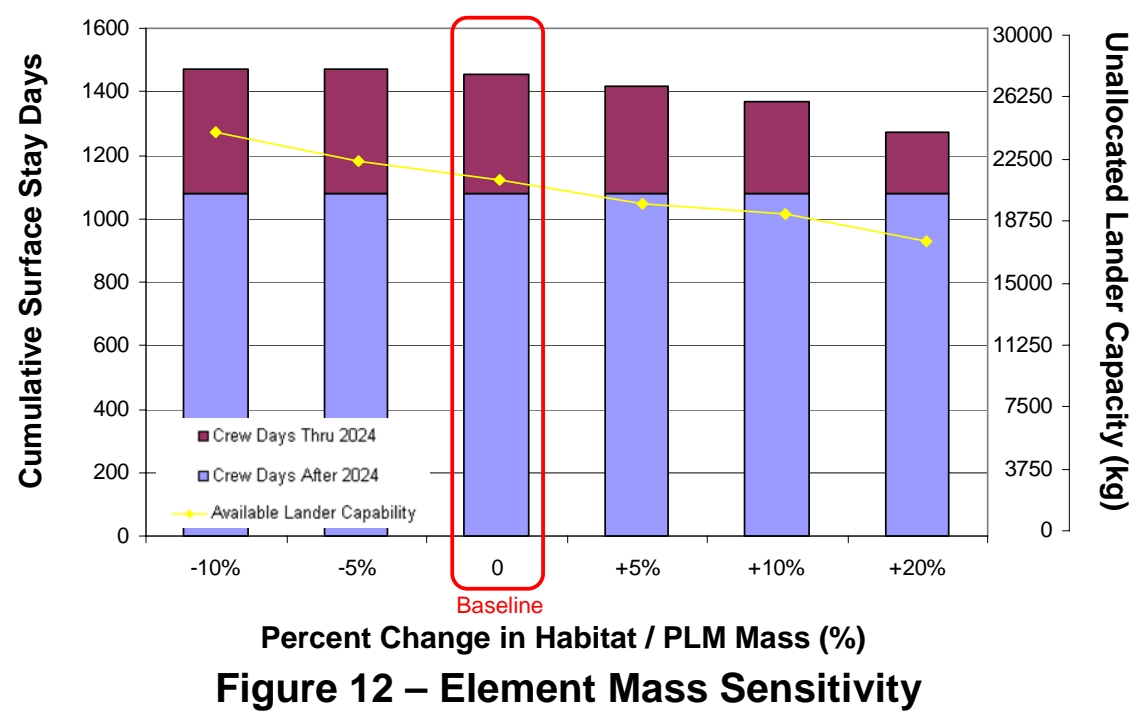

equipment must be delivered to the Outpost to support the elements. The sensitivity for spares and maintenance is varied from the original spares and maintenance mass assumptions by increments and decrements of 10 percent, 25 percent, and 50 percent. The masses of the element were not varied for this sensitivity.

Figure 11 shows a slight increase in crew surface stay duration and a significant increase in available lander capacity when the required spares and maintenance mass is reduced. Small increases in the required mass leads to a marginal decrease in crew surface stay duration and a significant reduction in available unallocated lander capacity, whereas large increases affect the crew surface stay duration considerably.

\section{Element Mass}

Closure for a flight depends mainly on lander mass and pressurized mass capability. Generally, because the focus of the scenarios is an early outpost buildup, the surface days earlier in the scenario are more likely to be affected by element mass. The more massive elements reduce the available mass for other elements/logistics that may be necessary to support human presence. Mass growth can cause the Outpost buildup phase to be extended and can delay the point at which sufficient functionality exists on the lunar surface to support crew operations.

Higher levels of water recovery start to increase unallocated lander capacity but do not increase crew surface duration.

The technologies assumed for this sensitivity analysis start to lead to diminishing returns and therefore the unallocated lander capacity increases because the need to bring water on the flights is no longer required to support the crew. Unfortunately, these technologies do not increase early mission crew surface stay duration. These missions do not have the mass or pressurized mass available to increase the days because other crew logistics, such as food and other consumables not directly related to water, are required.

\section{Spares and Maintenance}

The lunar architecture elements require repairs and replacements in order to run efficiently throughout the lifetime of the scenario. The assumption is that for each year a specified amount of spares and maintenance
The sensitivity analysis presented focuses on changes to the most massive elements: the habitat and the PLMs. The original element masses were varied both positively and negatively by 5 percent, 10 percent, and 20 percent. Performing this sensitivity analysis shows the impact that a major change to the mass of these elements will have on the entire scenario.

Variations in the habitat and the PLM mass from the baseline have a minimal impact on surface stay duration until the mass starts to increase significantly; at this point, the duration of the crew surface stay is reduced as seen in Figure 12. A slight impact is also noted on the available unallocated lander capacity.

\section{Logistics Carrier Capacities}

Logistics carriers are used to deliver pressurized, unpressurized, gas, and liquid logistics, with the mass of 


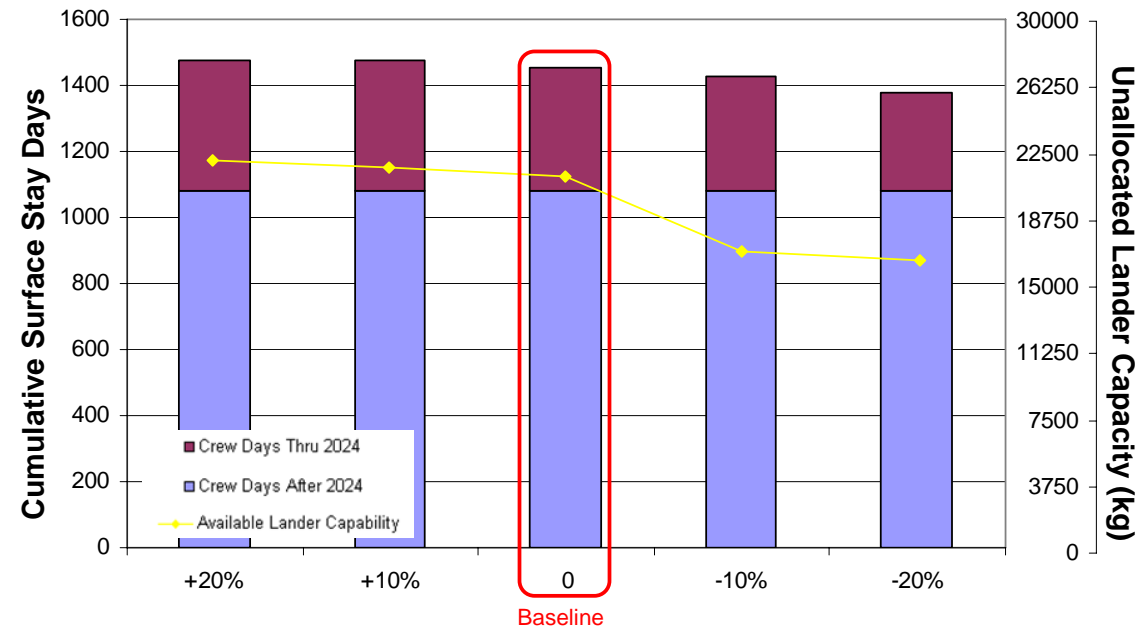

Percent Change in Logistics Carrier Capacity (\%)

Figure 13 - Logistics Carrier Capacities Sensitivity

the carriers typically accounting for 25 to 33 percent of the total delivered mass in the current scenarios. Varying the capacity of the carriers in 10 percent decrements and increments provides an assessment of the robustness of the baseline scenario to small changes in carrier capacity.

Only slight increases in crew surface duration and unallocated lander capacity are realized when the carrier capacity increases, as seen in Figure 13. Decreasing the capacity leads to a loss in crew surface stay duration and unallocated mass. The logistics carrier capacities drive the scenario primarily because of the change in available pressurized capacity.

\section{SUMMARY}

As NASA prepares to return to the Moon, lunar exploration architecture scenarios are being formulated to determine the best approach to satisfying the goal of continuous human presence on the lunar surface. Although a final detailed approach will not be decided for many years, strategic analysis can be utilized to aid decision makers in the comparison of approaches. The current lunar exploration architectures are focused on facilitating the early buildup of an Outpost, transporting a crew of four to the lunar surface, and maintaining continuous human presence. Given these objectives, strategic analysis has determined a handful of key drivers that significantly impact the performance, cost, risk, and value of the scenarios.

The five key drivers that significantly impact the architecture scenarios consist of inclusion of a cargo lander, mission rate, mission location, fixed-versusvariable cost/return on investment, and probabilistic manifesting. The inclusion of a cargo lander protects against the dependence of the success of a lunar exploration architecture on the crewed lander capacity to not decrease. The cargo lander increases the robustness of a scenario by allowing for the delivery of elements whose mass is greater than the crewed lander cargo capacity, facilitating the buildup of an Outpost, and delivering logistics in large quantities to support the goal of continued human presence. Although changes in mission rate drive the costs of the scenarios, the optimum ratio of crewed to cargo missions allows for meeting the aforementioned objectives while balancing the number of crew delivered to the lunar surface and the logistics and elements required to support the crew while on the surface. As determined by the NRC, science objectives are better met by visiting a variety of locations on the lunar surface. If achieving science objectives is highly important, then opportunities exist to trade Outpost crewed missions for sortie missions. For any of the architectures to be achieved, a "fixed" cost exists for even the minimum number of missions. As more crewed and cargo flights are added, this variable cost will increase the value of the scenarios; however, the risk will also be increased. In addition, in analyzing architecture scenarios, one must look at both planned, or deterministic, and expected, or probabilistic, results. The probabilistic analysis accounts for the uncertainty and risks that exist with human space flight.

Sensitivity analysis is also important for understanding how variables affect the scenarios and for determining whether these variables are key drivers. Sensitivity analysis, generally studied by the strategic analysis team, includes variability to mass, pressurized volume, lander capacity, and technology options. Many other variables can be studied to determine their importance to influencing the architecture scenarios. The SAT looks at cumulative surface stay days and unallocated lander capacity as the primary and secondary metrics for comparing scenarios in the performance of sensitivity analysis. 


\section{REFERENCES}

[1] "Exploration Systems Architecture Study -- Final Report”, NASA-TM-2005-214062, November 2005. url:

http://www.nasa.gov/mission_pages/constellation/news/ ESAS report.html

[2] William Cirillo, Kandyce Goodliff, Kevin Earle, J.D. Reeves, Chel Stromgren, Mark Andraschko, R. Gabe Merrill, "Strategic Analysis Overview," AIAA-20087778, 2008.

[3] William Cirillo, Kevin Earle, Kandyce Goodliff, J.D. Reeves, Mark Andraschko, R. Gabe Merrill, Chel Stromgren, "Analysis of Logistics in Support of a Human Lunar Outpost," Proceedings of the International Workshop on Modeling and Applied Simulation, Campora San Giovanni, Amantea (CS), Italy, 2008.

[4] Mark Andraschko, R. Gabe Merrill, Kevin Earle, "Logistics Modeling for Lunar Exploration Systems," AIAA-2008-7746, 2008.

[5] Doug Cooke, "Exploration Strategy and Architecture,” National Aeronautics and Space Administration, 2006. url:

http://www.nasa.gov/pdf/164310main_2nd_exp_conf_2 6_ExplorationStrategyArch_IntegratedLAT_CookeVol osinLavoie.pdf

[6] Doug Cooke, "Lunar Architecture Update," National Aeronautics and Space Administration, 2007. url: http://www.nasa.gov/pdf/190083main_AIAA_ESMD_f inalSPACE2007.pdf

[7] The Scientific Context for Exploration of the Moon, National Research Council of the National Academies, The National Academies Press, Washington, D.C., 2007.

[8] R. Gabe Merrill, Mark Andraschko, Chel Stromgren, William Cirillo, Kevin Earle, Kandyce Goodliff, "A Comparison of Probabilistic and Deterministic Strategic Analysis for Human Space Exploration”, AIAA-20087748, 2008.

\section{BIOGRAPHY}

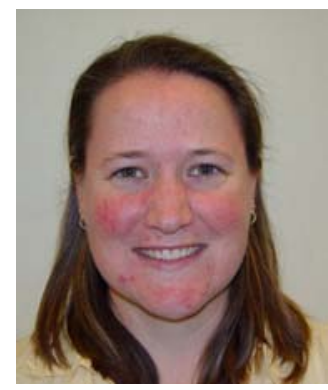

Kandyce Goodliff is an aerospace engineer at NASA Langley Research Center in Hampton, VA, with the Space Mission Analysis Branch (SMAB). Although she has been employed by NASA since 2007, she has supported SMAB since 1999 as a contractor working for various companies, with the most recent being Analytical Mechanics Associates, Inc., in Hampton, VA. She has participated in multiple conceptual design activities during her employment. Her primary roles as a systems analyst for SMAB are conceptual design and sizing of human and robotic spacecraft, mission and spacecraft analysis, and campaign analysis for human exploration.

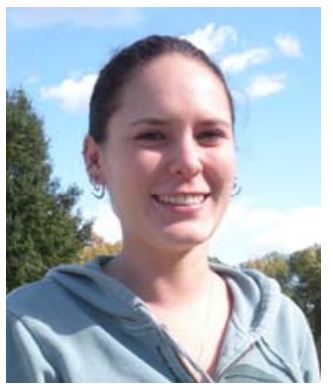

Hilary Shyface is a staff engineer with Analytical Mechanics Associates, Inc., in Hampton, Virginia. Ms. Shyface has performed campaign analysis in support of NASA's Exploration System Mission Directorate and Constellation Program. Prior to her current position, Ms. Shyface earned a Bachelor of Science in Mechanical Engineering from the University of Nevada, Las Vegas.

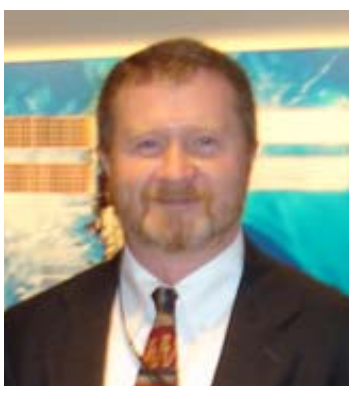

William Cirillo currently serves as a senior researcher at NASA Langley Research Center in Hampton, VA, where he has worked for past 20 years in the area of Human Space Flight Systems Analysis. His work has included studies of Space Shuttle, International Space Station, and Human Exploration beyond low Earth orbit. In 2005, Mr. Cirillo served at NASA Headquarters as a core member of the Exploration Systems Architecture Study (ESAS) team, where he was responsible for studying the use of Ares I/Orion in meeting future ISS crew and logistics transportation needs. Mr. Cirillo currently leads a team of analysts within the NASA Constellation Program in assessing at a strategic and tactical level the manifesting of assembly and logistics flights for establishing a permanent human presence on the Moon.

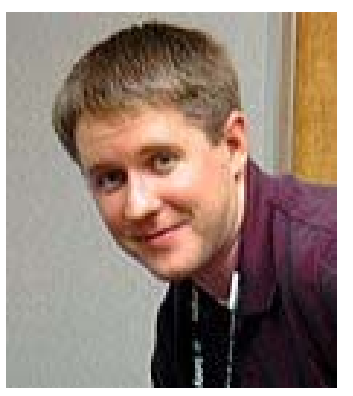

Kevin Earle is an aerospace engineer in NASA Langley Research Center's Space Mission Analysis Branch. Since 2003, he has provided spacecraft design, systems analysis, and software development support to NASA's Exploration System Mission Directorate (ESMD) and Science Mission Directorate. Over the past three years he has been a member of the NASA ESMD and the Constellation Program Strategic Analysis Team, 
providing scenario manifesting and software development support for the Lunar Architecture Team and the Constellation Architecture Team.

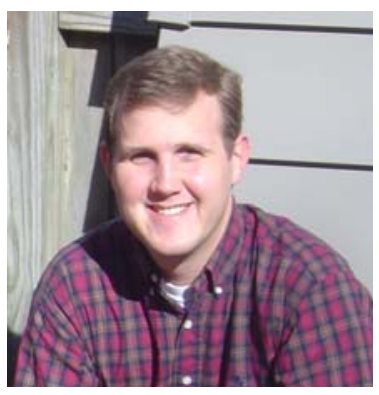

J. D. Reeves is an aerospace engineer in the Vehicle Analysis Branch at NASA Langley Research Center and has provided systems engineering expertise in the areas of affordability and cost estimating, concept of operations analysis, technology assessment, and general architecture integration since 2004. His current and recent responsibilities include co-leading cost assessment and affordability integration teams with a focus on lunar surface systems and architectures. He has previously worked for the National Institute of Aerospace and BearingPoint, Inc. Mr. Reeves holds a Bachelor of Science degree in Industrial and Systems Engineering and a Master of Science degree in Aerospace Engineering from the Georgia Institute of Technology.

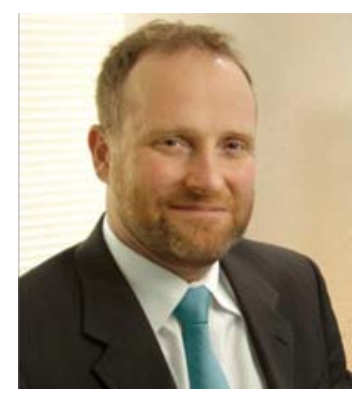

Chel Stromgren currently serves as the chief scientist of the Center for Strategic Analytics at Science Application International Corporation (SAIC). In this role, Mr. Stromgren leads the development of probability and risk-based strategic models and strategic analysis of complex system development. Mr. Stromgren has supported NASA in the analysis of Space Shuttle and International Space Station operations in the post-Columbia environment and has led the development of strategic campaign models for the lunar exploration initiatives. He holds a Bachelor of Science degree in Marine Engineering and Naval Architecture from the Webb Institute and a Master of Science degree in Systems Management from the Massachusetts Institute of Technology.

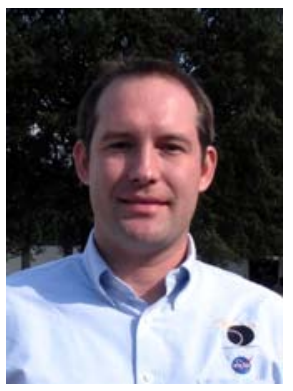

Gabe Merrill is a project engineer with Analytical Mechanics Associates, Inc. Since 2001, he has supported integrated analysis capability development for launch vehicle and space transportation sizing and trajectory development at NASA Langley Research Center. Over the past three years he has been a member of the NASA

Exploration Systems Mission Directorate and the Constellation Program Strategic Analysis Team, providing scenario manifesting and capability development support for the Lunar Architecture Team and the Constellation Architecture Team.

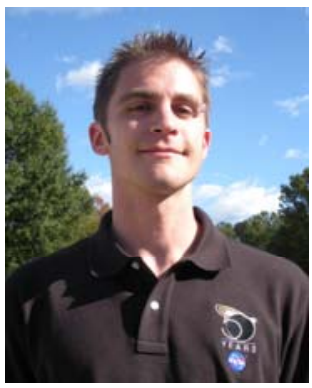

Mark Andraschko is a project engineer with Analytical Mechanics Associates, Inc., in Hampton, VA. For the past two years, he has performed campaign analysis, spacecraft systems analysis, and software development in support of NASA's Science Mission Directorate and Exploration System Mission Directorate. Prior to this, Mr. Andraschko earned a Masters of Science degree in Engineering Mechanics from the University of Wisconsin-Madison, performing cryogenics research in support of the ITER fusion reactor program and performed modeling and simulation studies for the F-35 Program.

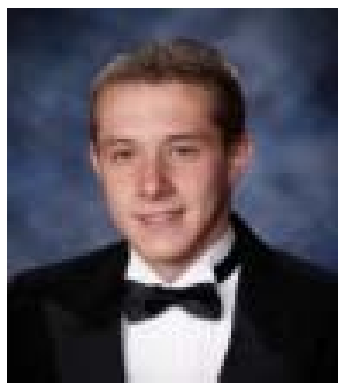

Christopher Cirillo is a senior at Warwick High School, where he is a member of the International Baccalaureate Program. During the summer of 2008, he volunteered at NASA Langley Research Center where he performed campaign analysis. 